\title{
A homogenization method for non variational problems
}

\author{
Luis Caffarelli
}

\begin{abstract}
We would like to discuss in these lectures three problems of homogenization and their interplay. They are:

A) The construction of plane-like solutions to the minimal surface equation in periodic media.

B) Pulsating wave solutions to a combustion problem and its homogenization limit.

C) Existence of homogenization limits for solutions to fully linear equations in ergodic random media.

We will try to point out what the main techniques are, and their common aspects.
\end{abstract}

Part 1) The construction of plane-like solutions to periodic minimal surface equations.

In two dimensions, minimal surfaces are just geodesics: we are given in $R^{2}$ a differential of length $a(x, \vec{\nu})$ and given two points $x, y$, we want to minimize

$$
d(x, y)=\inf L(\gamma)=\int_{\gamma} a(z, \vec{\sigma}) d s
$$

among all curves $\gamma$ joining $x$ to $y$.

Here $s$ is the usual differential of length, $\sigma$ the unit tangent vector. The function $a(x, \sigma)$ is periodic in $x$, strictly positive $(0<\lambda \leq a(x, \sigma) \leq$ $\Lambda$ ) and, to avoid the formation of Young measures (that is: oscillatory zig-zags) when trying to construct geodesics, it must satisfy

$$
\text { " }|v| a\left(x, \frac{v}{|v|}\right) \text { is a strictly convex cone." }
$$

This is a "classical" condition of ellipticity for area minimizers, and the regularity of solutions has been studied, for instance by Schoen and Simon.

The author was supported in part by NSF grants. 
Let's assume that $a$ is smooth although this property is not needed. Due to the periodicity, at large distances $d(x, y)$ becomes "almost" translation invariant, since for any $z$

$$
|d(x+z, y+z)-d(x, y)| \leq 2 \sqrt{n} \Lambda .
$$

(All you need to compare them is to travel the length of one period, since geodesics are invariant by integer translations.)

Another way of saying the same thing is to look at the geodesics from very far away, that is to rescale the medium by $\varepsilon$ very small

$$
a_{\varepsilon}(x, \vec{\sigma})=a\left(\frac{x}{\varepsilon}, \vec{\sigma}\right) .
$$

Then finite geodesics become almost translation invariant

$$
\left|d_{\varepsilon}(x, y)-d_{\varepsilon}(x+z, y+z)\right| \leq 2 \varepsilon \sqrt{n} \Lambda
$$

and as $\varepsilon$ goes to zero we obtain an "effective norm,"

$$
\|x\|=\lim _{\varepsilon \rightarrow 0} d_{\varepsilon}(x, 0) .
$$

Always in 2 dimensions, the question we are interested in studying is the following: Given any line, say

$$
L=\{\lambda \sigma, \lambda \in R\},
$$

can we construct a global geodesic, $S$, that stays at finite distance from $L$ ? That is $S$ remains trapped in a strip, around $L$ whose width depends only on $\lambda, \Lambda$. (See Figure 1.)

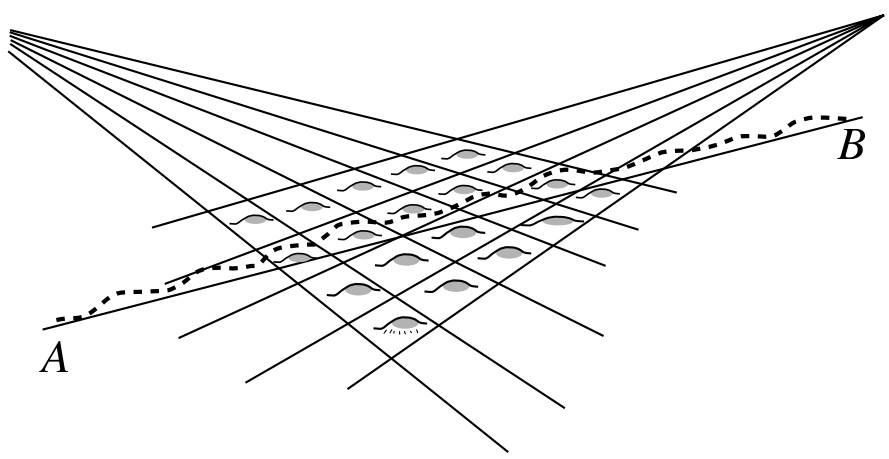

Figure 1. "Line like geodesic".

The answer is yes in $2-d$ (Morse [8]) and no in $3-d$ (Hevlund [7]). An inspection of Hevlund counterexample shows that, at a difference with classical homogenization, where diffusion processes tend to "average the medium", geodesics try to "beat the medium" by choosing specific paths, and leaving "bad areas" untouched (see Figure 2).

The interest of constructing "line-like" geodesics was related to foliating the torus with them (or at least "laminate" it, that is not fully cover it). 


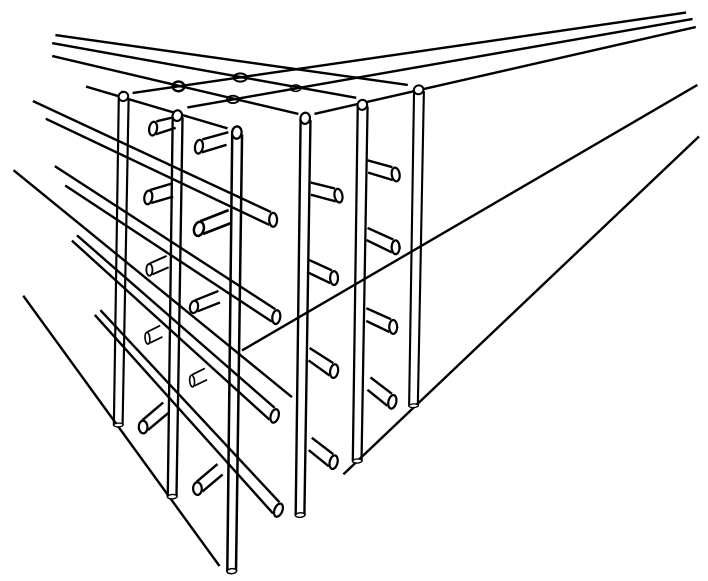

Figure 2. Hevlund Counterexample: It costs "one" to travel inside narrow pipes, $M$ outside. Then, the best strategy is to jump only once from pipe to pipe, i.e., the effective norm is $\|x\|=|x|+|y|+|z|$.

In the early 80's Moser [9] suggested that in $R^{n}$, at a difference with geodesics, minimal hypersurfaces should be forced to "average" the medium, and given any plane $\pi$, it should be possible to construct "plane-like" minimal surfaces for the periodic medium.

More precisely, given a "differential of area" we would like to consider "surfaces" $S$ that locally minimize

$$
A^{*}(S)=\int_{S} a(x, \nu) d A
$$

where $d A$ is the usual differential of area $\left(H^{n-1}\right), \nu$ the normal vector to $A$, and $a$, as before satisfies,

a) $0<\lambda \leq a(x, \nu) \leq \Lambda$;

b) $|v| a(x, v /|v|)$ is a strictly convex cone;

c) $a$ is periodic in $X$.

THEOREM 1. There exists a universal constant $M(\lambda, \Lambda, m)$ such that: for any unit vector $\nu_{0}$ there exists an $A^{*}$ local area minimizer $S$ contained in the strip $\pi_{M}=\left\{x:\left|\left\langle x, \nu_{0}\right\rangle\right|<M\right\}$. (See Figure 3.)

Let me explain the terms in the theorem.

We will work in the framework of boundaries of sets of locally finite perimeter.

Roughly, a set of locally finite perimeter $\Omega$ is the limit of polyhedra, $\Omega_{k}$, of locally finite area (i.e.,

$$
\left|\left(\Omega \Delta \Omega_{k}\right) \cap B_{M}\right| \rightarrow 0
$$




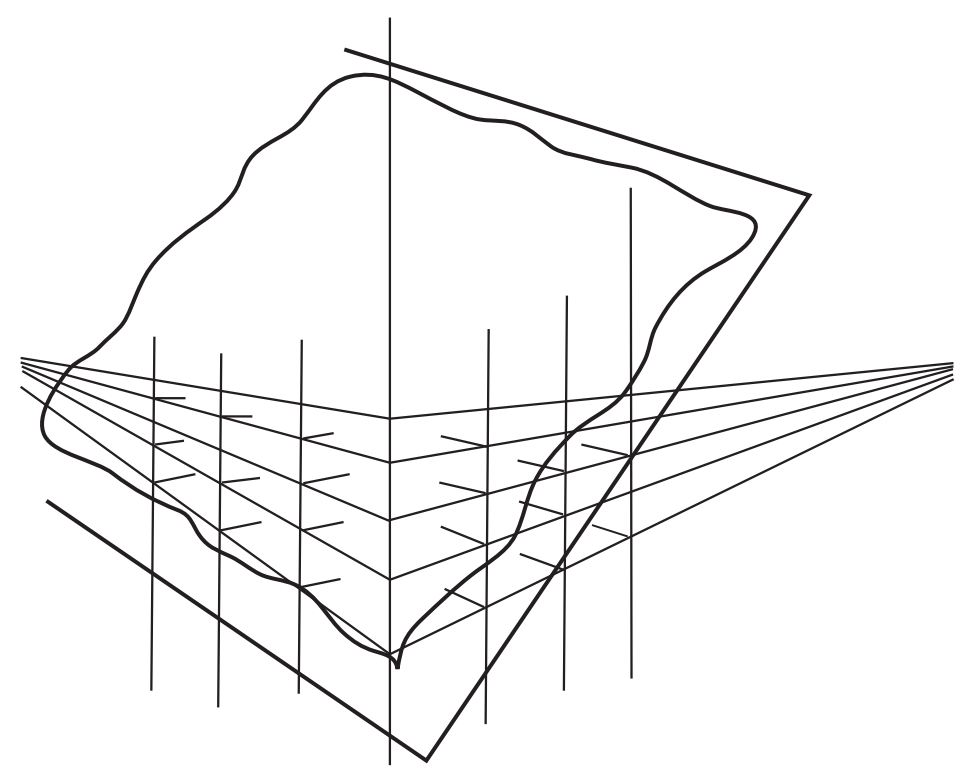

Figure 3. Plane-like minimal surface in a periodic medium (for instance, a medium with a periodic Riemann metric).

for any ball $B_{M}$, and Area $\left(\partial \Omega_{k} \cap B_{M}\right) \leq C\left(B_{M}\right)$ for all $\left.k\right)$. In principle we can define the "area measure" of $\partial \Omega$ weakly, using the divergence theorem:

For any smooth vector field $y(x)$

$$
\text { " } \int_{\partial \Omega} y(x) \cdot \nu d A "=\int_{\Omega} \operatorname{div} y d x
$$

and $\partial \Omega$ is of "finite perimeter" (i.e., finite total area), if

$$
\left|\int_{\Omega} \operatorname{div} y d x\right| \leq \sup _{\Omega}|y| .
$$

This definition was introduced by Cacciopoli.

It turns out (see, for instance, Guisti) that the notion of normal vector $\nu$, and $d A$ are well defined, and the structure of the boundary of a set of finite perimeter well understood. Under our hypothesis $A^{*}$, then, makes sense and is lower semicontinuous under convergence in measure for sets.

\section{Main steps of the proof.}

We will consider the family of sets $\mathbb{D}$ such that $\Omega \in \mathbb{D}$ if $\Omega$ is of locally finite perimeter and:

$$
\begin{aligned}
& \pi_{M}^{-}=\left\{x:\left\langle x, \nu_{0}\right\rangle \leq-M\right\} \subset \Omega \subset \\
& \pi_{M}^{+}=\left\{x:\left\langle x, \nu_{0}\right\rangle \leq M,\right.
\end{aligned}
$$


and further, within $\mathbb{D}$, those sets $\Omega_{0}$ that are local $A^{*}$-minimizers among sets $\Omega \in \mathbb{D}$. (See Figure 4.)

Of course, such an $\Omega_{0}$ is not a "free" local minimizer since whenever $\partial \Omega_{0}$ touches the boundary of $\pi^{-}$or $\pi^{+}$we are not free to perturb it "outwards".

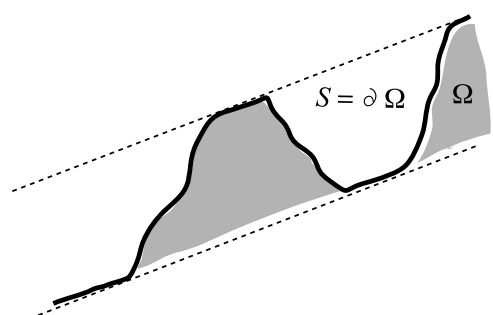

FIGURE 4. Restricted Minimizer.

Our objective is to show that if $M$ is large enough $S_{0}=\partial \Omega_{0}$ does not see this restriction.

The main ingredients are:

a) a positive density property (see Figure 5): Depending only on $\lambda, \Lambda$ a minimizer, $\partial \Omega_{0}$ of $A^{*}$ satisfies:

Let $x_{0} \in \partial \Omega_{0}$, then

$$
\begin{gathered}
0<C_{0} \leq \frac{\left|\Omega_{0} \cap B r\left(x_{0}\right)\right|}{|B r|} \leq C_{1}<1 \\
C_{0} r^{n-1} \leq A^{*}\left(\partial \Omega_{0} \cap B r\right) \leq C_{1} r^{n-1} .
\end{gathered}
$$

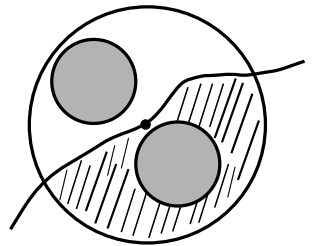

Figure 5. Positive density property.

Property a) tells us that for large balls $B_{T}(0)$, the number $N$ of disjoint unit cubes intersecting $\partial \Omega_{0}$ must be of order $N \sim C_{1} T^{n-1}$ independently of $M$.

Since the strip $\pi_{M} \cap B_{T}$ has $\sim M T^{n-1}$ cubes, many cubes in $\pi_{M} \cap B_{T}$ must be contained in $\Omega_{0}$ or $\mathcal{C} \Omega_{0}$.

b) Minimizers are "ordered," (see Figure 6) that is if $\partial \Omega_{0}, \partial \Omega_{1}$ are minimizers, then $\partial\left(\Omega_{0} \cup \Omega_{1}\right)$ and $\partial\left(\Omega_{0} \cap \Omega_{1}\right)$ are minimizers.

Therefore we may consider the "smallest" minimizer:

$$
\Omega=\bigcap \Omega_{\alpha}
$$




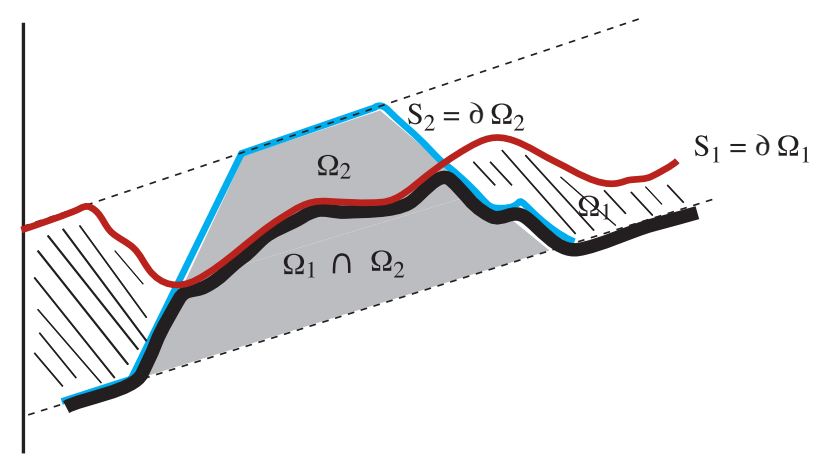

Figure 6. Minimizers are "ordered".

with $\Omega_{\alpha}$ in the family of all admissible minimizers. (We point out that, although the problem is variational, the selection of the "special solution" is done through a Perron-like method.)

Such a choice of $\bar{\Omega}$ allows us to recuperate an important property, the Birkhoff property: If $\tau$ is an integer translation with $\left\langle\tau \cdot \nu_{0}\right\rangle \leq 0$ (resp. $\geq 0$ ) then (see Figure 7 )

$$
\tau(\bar{\Omega}) \subset \bar{\Omega} \quad(\text { resp. } \supset \bar{\Omega}) .
$$

Indeed:

$$
\tau(\bar{\Omega}) \cap \bar{\Omega} \text { and } \tau(\bar{\Omega}) \cup \bar{\Omega}
$$

are minimizers respectively for $\tau\left(\pi_{M}\right)$ and $\pi_{M}$, while $\bar{\Omega}$ and $\tau(\bar{\Omega})$ are the actual "smallest" minimizers.

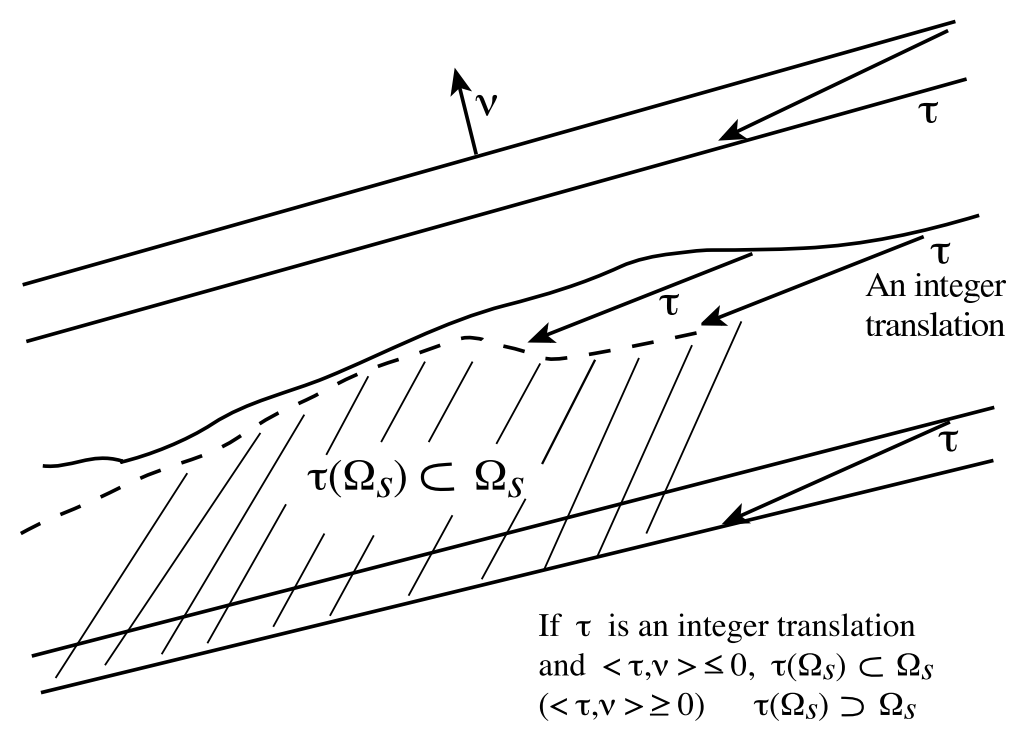

FiguRE 7. Birkhoff Property. Integer translations send $\tau(\bar{\Omega})$ inside $\bar{\Omega}$ (or) $\bar{\Omega}$ inside $\tau\left(\Omega_{1}\right)$ depending if $\left\langle\tau, \nu_{0}\right\rangle \leq 0$ or $\left\langle\tau, \nu_{0}\right\rangle \geq 0$. 
This makes $\partial \Omega$ "almost" translation invariant, in fact translation invariant if we are willing to neglect one period. Combining these two facts, we see that if $M$ is large,

i) there are many clean cubes since:

a) The area of $\partial \Omega$ is bounded by the area of the bottom plane (a competing surface).

b) The number of cubes intersecting $\partial \Omega$ is bounded by the area of $\partial \Omega$ (from the density properties above).

c) The total number of cubes is $M$ times the area of the bottom.

ii) If $Q$ is a "clean cube" inside $\Omega$ (resp. inside $\mathcal{C} \Omega$ ), then any integer translation with $\left\langle\mathcal{T}, \nu_{0}\right\rangle \leq 1$ (resp. $\left.\left\langle\mathcal{T}, \nu_{0}\right\rangle \geq 1\right)$ sends $Q$ into another clean cube, because of the Birkhoff property.

It follows that, for $M$ large, $S=\partial \bar{\Omega}$ is far from the top, and may touch the bottom but is still a free minimizer (see Caffarelli-Llave [4] for details, also Auer and Bangert for related work).

\section{Part 2) Flame Propagation.}

The second homogenization problem that we would like to discuss concerns the effective speed of pulsating waves in problems of flame propagation.

In the homogeneous case, the problem consists in solving for $u$ the following evolution problem, $P_{0}$ :

$$
\Delta u-u_{t}=\lambda \beta_{\delta}(u)
$$

with initial data

$$
u_{0}(x, 0)=g\left(x_{0}\right) \text { prescribed. }
$$

The function $u$ (and thus $g$ ) is expected to be between 0 and 1 .

The non-negative function $\beta_{\varepsilon}$ has support concentrated near $u=0$, and as $\delta$ goes to zero, it approximates a Dirac's delta sitting on $u=0$. In fact, in the limit $\delta=0$ we have generically the following configuration: There is a set, $\Lambda$, where $u \equiv 0$, on its complement $\Omega, u>0$ and satisfies the heat equation, $\Delta u-u_{t}=0$ along $\partial \Omega, u_{\nu}^{+}=\lambda$.

As with the case of minimal surfaces above, to understand how a flame front propagates one focuses on special solutions or profiles called "traveling wave solutions."

These are profiles that, in some system of coordinates, depend on $x_{1}-c t$. In other words the profile (the flame configuration) travels with speed $c$ in the direction $e_{1}$. For the homogeneous case they are simply

$$
T_{c}=\left(1-e^{-c x-c^{2} t}\right)^{+}
$$

and their translations (see Figure 8). That is, the speed of propagation $c$ is given by $\lambda$. We are interested in the case in which $\lambda$ is replaced by a periodic coefficient $f(x)$. 


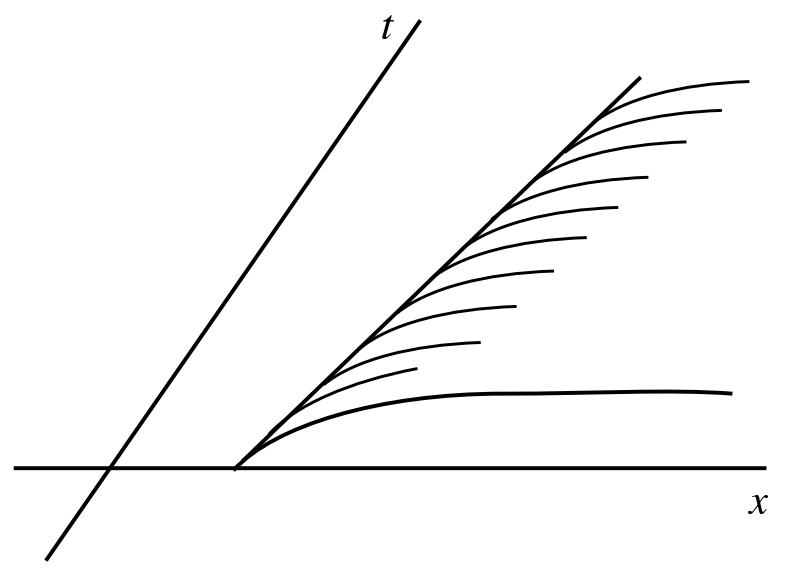

FiguRE 8. Travelling wave profile $\left(1-e^{-(x-t)}\right)^{+}$.

If the oscillation of $f$ happens at scale epsilon, the problem $P_{\varepsilon}$ has a slightly different profile, with some unknown speed $c$. In this case, the profile corresponding to plane-like minimal surfaces are called "pulsating wave" solutions. That is, we look for solutions to the problem

$$
\Delta u-u_{t}=f\left(\frac{x}{\varepsilon}\right) \beta_{\delta}(u)
$$

trapped between two traveling waves with the same speed of propagation, $T_{c}$ and a translation $T_{c}(\cdot-M)$. The constant $c$, the effective speed of propagation, is determined by $f, \varepsilon$ and $\delta$. (This replaces the strip $\pi_{M}$ in the case of minimal surfaces.) (See Figure 9.)

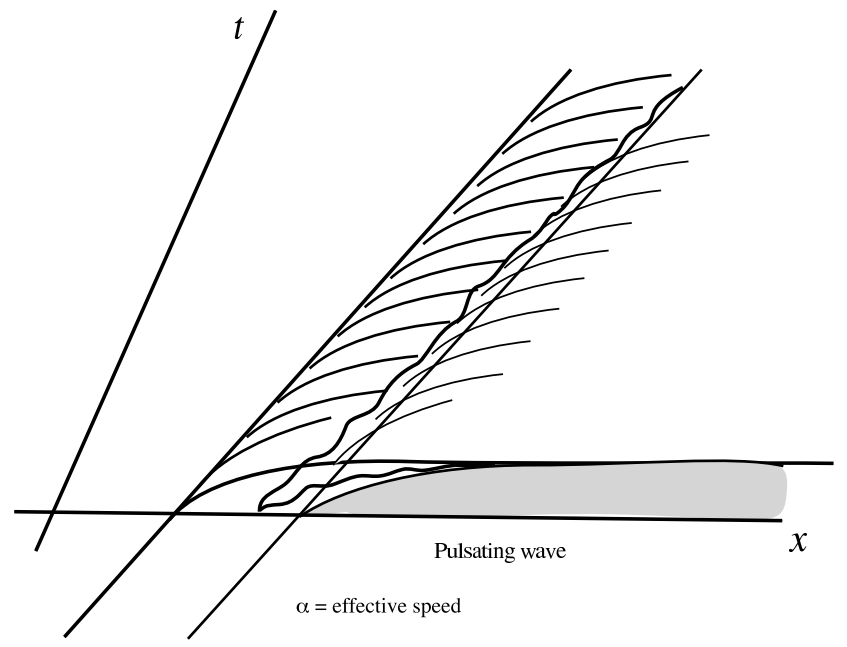

Figure 9. Pulsating wave, trapped between two translations of the same travelling wave. The travelling wave is not arbitrary. It is uniquely defined and gives us the "effective" speed of propagation. 
Notice that here we have preserved the two scales, $\varepsilon$ and $\delta$, and we are interested in the different ways they tend to zero.

In particular we will assume $\delta<\varepsilon$, since for $\varepsilon$ going to zero faster than $e$ the problem converges to the homogeneous case.

TheOREM 2. We assume that $0<\lambda \leq f(x) \leq \Lambda$ and that $f$ is Lipschitz. Given any unit vector e, there exists a unique pulsating wave in the direction e. Its effective speed $c$ is bounded by $\lambda$ and $\Lambda$ and the separation $M$ is

$$
M \leq M_{0} \cdot \varepsilon
$$

with $M_{0}(\lambda, \Lambda)$.

In this (simple) model, it is assumed that gas ignites when the temperature $T$ attains a critical (ignition) temperature $\theta_{i}$.

Ahead of the flame, temperature diffuses (a convection term is also present) and behind the flame the temperature remains $\theta_{i}$. On the ignition band there is a heat source $\theta_{\delta}(T)$. The parameter $\delta$ indicates the width of the range of temperatures $\sim\left(\theta_{i}-\delta, \theta_{i}\right)$, on which ignition takes place. We describe the problem mathematically for $u=\frac{\theta_{i}-\theta}{\theta_{i}}$. (T at infinity is $\theta_{\infty}=0$.)

If we let $\delta$ go to zero first (i.e., we have the sharp transition case with $u_{\nu}=f(x / \varepsilon)$ ) and then let $\varepsilon$ go to zero, the effective speed $c$ is given

i) In 1 dimension by the minimum of $f$.

ii) In $n$ dimensions by the slope at infinity of the solution of the stationary problem with minimal slope.

The smallest possible effective slope of the stationary problem: See the work of Berestycki and Hamel [11] for the existence of pulsating waves, Caffarelli, Lee and Mellet [12] for the homogenization limits.

To understand this statement let us look at the sharp stationary problem first:

In one dimension, in the interval $[0,1]$, we look for solutions of $u_{x x}=$ 0 when $x>0$, and when $u$ becomes zero we want $u_{x}=f(x / \varepsilon)$, for instance $f(x / \varepsilon)=3 t \cos \frac{x}{\varepsilon}$. Of course, when $u>0$, it is linear.

If we prescribe $u(1)=1$, and $u$ becomes zero at $x_{0}$, its slope is $\sigma(u)=\frac{1}{\left(1-x_{0}\right)}$.

Thus we have to match

$$
\frac{1}{1-x_{0}} \text { with } 3 t \cos \frac{x_{0}}{\varepsilon}=f\left(\frac{x_{0}}{\varepsilon}\right) \text {. }
$$

Since $2 \leq f \leq 4$, we see that all matches take place for $\frac{1}{2} \leq x_{0} \leq \frac{3}{4}$ and in fact, for $\varepsilon$ giving to zero they fill the interval $\left[\frac{1}{2}, \frac{3}{4}\right]$; thus any slope within $\frac{1}{2}$ and $\frac{3}{4}$ is an "effective speed". (See Figure 10.)

But we can distinguish three values

$$
x_{0}=1 / 2\left(\text { the largest solution, } u_{1}\right)
$$




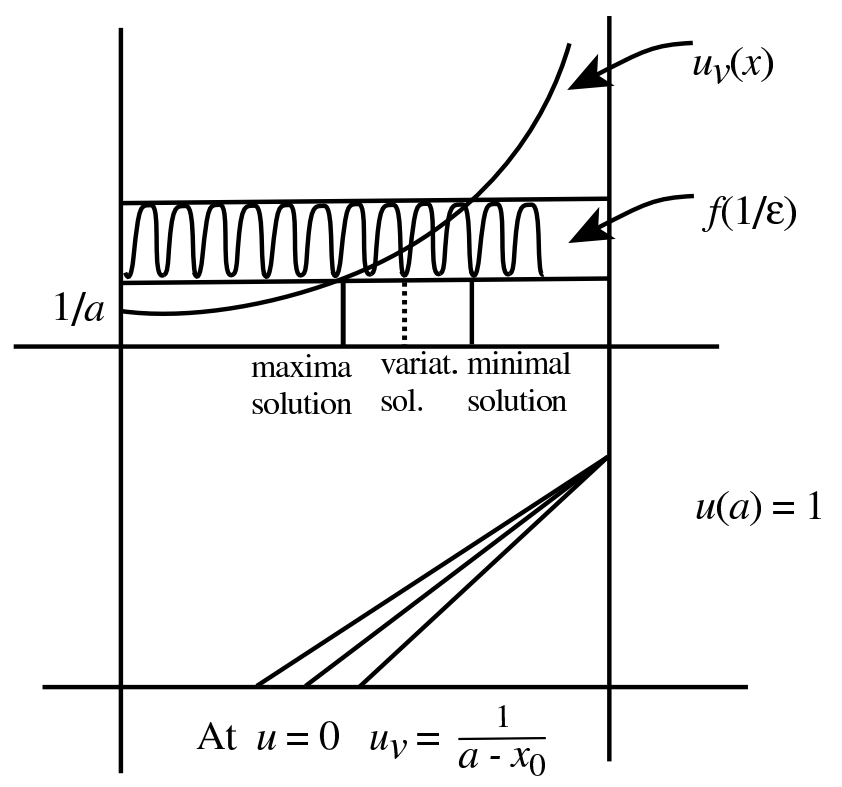

FIgURE 10. There are many solutions to the free boundary problem: $u\left(x_{0}\right)=0, u_{\nu}\left(x_{0}\right)=f\left(\frac{x_{0}}{\varepsilon}\right)$.

$x_{0}=3 / 4$ (the least supersolution, $u_{2}$ ) and

the variational solution $u_{3}$

$u_{3}^{\varepsilon}$ minimizes

that corresponds to

$$
\int\left(\nabla u_{3}^{\varepsilon}\right)^{2}+\left(\sqrt{f_{\varepsilon}}\right) \chi_{u_{3}^{\varepsilon}>0}
$$

$$
\frac{1}{1-x_{0}}=\left(f \sqrt{f_{\varepsilon}}\right)^{2} .
$$

Although the variational solution is the appropriate one in many instances, the least supersolution will trap the solution of any evolution process where $\Omega_{t}=\{u(x, t)>0\}$ is expanding while the largest subsolution will trap contracting $\Omega_{t}$ 's which is our case.

Although in general effective constants in homogenization are difficult to compute, in this case we found a surprising phenomena, suggested by numerical simulation.

In one dimension, as $\varepsilon$-decreased, we noticed that the edge of the pulsating wave lingered more and more in the points where $f$ realized its minimum and then jumped to the next minimum of $f$.

We prove that this is in fact the case, as stated above.

We see again here, the role of the "minimal" solution in the homogenization process: when a surface "travels through a periodic media," it seems to chose the "smallest" propagation speed. This can be verified for instance in the case of movement by mean curvature (Caffarelli and Lee) for the expansion or contraction of capillary drops (Caffarelli 


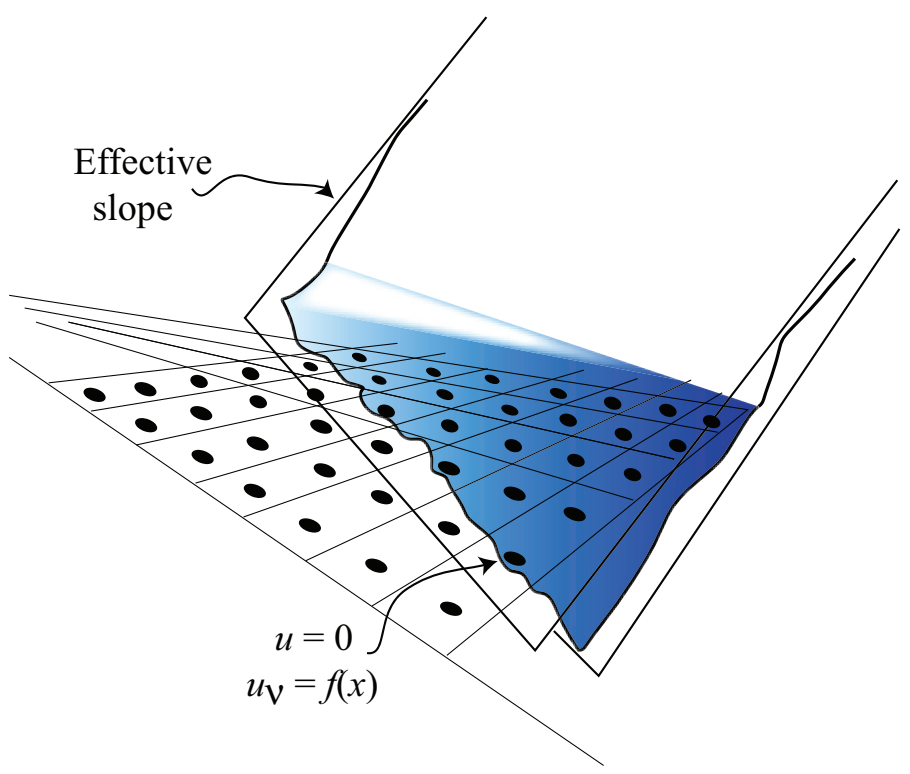

Figure 11. The "plane-like" solution with smallest slope at infinity.

and Mellet) that gives rise to hysteresis (the equilibrium shape of a contracting drop is different than an expanding one) that have been experimentally observed.

In higher dimensions, in order to find the effective speed of pulsating fronts, we must study plane-like stationary solutions. That is solutions of the stationary free boundary problem

$$
\left\{\begin{array}{l}
\Delta u=0 \text { in } \Omega=\{u>0\} \\
u_{\nu}=f(x) \text { along } \partial \Omega
\end{array}\right.
$$

that are trapped between two half planes:

$$
c\langle e, x\rangle^{+} \text {and } c\langle e, x-M e\rangle^{+}
$$

The problem is, though, that $c$ is not unique anymore.

For instance, if $f(x) \equiv 1$ in $Q_{1}$ except for a small ball $B_{\tau}(0)$ where $f$ is very large, and we extend it periodically, there will be rational directions for which we can completely avoid the lattice generated by $B_{\tau}$, and $c=1$ will be one solution (the minimal one), while the variational solution, with slope $(f \sqrt{f})^{2}$, will be another. Notice here again the phenomena where the free boundary may try to avoid averaging the medium, and the extremal solutions are relevant. (See Figure 11.)

The proof of Theorem 2 is somewhat intricate. We point out that the main ideas of the previous discussion on minimal surfaces reappear: 
A Birkhoff property (from uniqueness and the fact that the minimum of two solutions is a supersolution) and a positive density property for $\Omega$ at scale one (scale $\varepsilon$ for the problem).

We complete this discussion with the remark that, at least in 1dimension, when $f(x)$ is random instead of periodic, one can still prove that there is an almost surely effective speed, and it is given by the essential infimum of $f(x, \omega)$.

\section{Part 3) Existence of homogenization limits for fully non- linear equations.}

Let me start by giving a classical example of a fully non-linear equation. Suppose particles move randomly in a non-homogeneous domain $\Omega$ : At time $t_{k}$ a particle in position $X_{k}$ will move to a new position

$$
X_{k+1}=X_{k}+\varepsilon \sigma_{i j}(x) \chi
$$

where $\chi$ is chosen randomly (uniformly) from the unit sphere.

When a particle reaches $\partial \Omega$ it pays $g(x)$ and it is taken out.

If we want to find out the average payment $u(x)$ that a particle at position $x$ will eventually make, by realizing that $u(x)=\oint_{S^{1}} u(x+$ $t \sigma(x) \chi) d s$ we find that infinitesimally (for $t \rightarrow 0$ ) $u$ will satisfy

$$
\left\{\begin{array}{l}
L(u)=\mathcal{A}_{i j}(X) D_{i j} u=0 \\
u / \partial \Omega=g
\end{array}\right.
$$

with $\mathcal{A}_{i j}=\sigma^{T} \sigma$.

Suppose now that we are allowed to design the medium, (i.e., choose $\sigma(x))$ with the only constraint that

$$
I \leq \sigma \leq 2 I
$$

with the purpose of minimizing the cost $u(x)$. What is the optimal design? To find it, we must construct a solution to the following (fully non-linear) problem: Find a function $v$ such that $v / \partial \Omega=g(x)$ and at every point $X$, if $\lambda_{j}$ are the eigenvalues of $D^{2} v$,

$$
F\left(D^{2} v\right)=\sum_{\lambda_{j}>0} \lambda_{j}+\sum_{\lambda_{j}<0} 4 \lambda_{j}=0 .
$$

Indeed:

a) $v$ is a subsolution of any one of the admissible equations:

$$
\mathcal{A}_{i j} D_{i j} v \geq 0
$$

for any $I \leq \mathcal{A}_{i j} \leq 4 I$.

But, on the other hand

b) $v$ is in the class of admissible solutions. 
Indeed, if we rewrite the (fully non-linear) equation $F\left(D^{2} v\right)$ in a fixed system of coordinates, it will read

$$
\tilde{\mathcal{A}}_{i j}(x) D_{i j} v=0
$$

with $\tilde{\mathcal{A}}_{i j}(x)$ some matrix with eigenvalues one and four. Therefore $\tilde{\mathcal{A}}_{i j}$ provides the optimal design and $v$ the expected cost.

The operator above,

$$
F\left(D^{2} v\right)=\sum_{\lambda_{j}>0} \alpha \lambda_{j}+\sum_{\lambda_{j}<0} \beta \lambda_{j}
$$

is called a Pucci extremal operator.

In fact, $v$ can be described as the unique solution to

$$
F_{P}\left(D^{2} v\right)=\inf _{I \leq \mathcal{A}_{i j} \leq 4 I} \mathcal{A}_{i j}(x) D_{i j} v=0 .
$$

This is the typical form of a fully non-linear equation

$$
F_{A}\left(D^{2} w\right)=\inf _{L \in A} L\left(D^{2} w\right)=0
$$

where $L$ is a family of linear equations, or more generally

$$
F_{A, B}=\sup _{\beta \in B}\left(\inf _{\alpha \in A} L_{\alpha, \beta}\left(D^{2} w\right)\right) .
$$

Note that in the first case $F_{A}$ is a concave function of $D^{2} w$, an important structural property.

Finally, to motivate the randomness, suppose for instance that after construction the medium suffers a random deformation, due for instance to cooling.

The solution $v$ will now satisfy a new equation, say

$$
\widetilde{F}_{\omega}\left(D^{2} v, x\right)=0,
$$

where the variable $\omega$ indicates the particular random deformation of the medium.

Nevertheless, if the deformation of the medium occurs at a scale much smaller than the size of $\Omega$ and has some "organization" that locally repeats itself, we could expect that in the large scale solutions of $\widetilde{F}_{\omega}\left(D^{2} v, x\right)$ converge to solutions $v_{0}$ of some equation

$$
F_{0}\left(D^{2} v_{0}\right)=0
$$

where both the dependence in $X$ and $\omega$ will disappear . That is, with $\omega$ in an appropriate probability space $(M, \mu)$ of "possible deformations", and $\varepsilon$ the scale of the deformation (with respect to $\Omega$ ), we expect that as $\varepsilon$ goes to zero, solutions $v_{\varepsilon}$ to

$$
F_{\omega}\left(D^{2} v_{\varepsilon}, \frac{x}{\varepsilon}\right)=0
$$


converge almost surely in $\omega$ to a solution $v_{0}$ of the "homogenized" operator

$$
F\left(D^{2} v_{0}\right)=0 .
$$

Let me start my trying to present the link of this topic with the previous one. Second order equations arise in two different fashions: in divergence form

$$
D_{i}\left(A_{i j}(x) D_{j}\right) u=0
$$

in continuous mechanics, calculus of variations, etc, and in non-divergence form

$$
A_{i j}(x) D_{i j} u
$$

in probability and optimal control.

These two types, although similar in many aspects, are essentially different. The first theory is based on functional analysis: Energy considerations, weak formulations, compensated compactness, etc. If $A_{i j}$ are, for instance, just Hölder continuous, it is impossible to exhibit a single super- or subsolution of such an equation. Its infinitesimal expression is just the consequence of a set additive conservation law that has been integrated by parts.

A non-divergence equation, in contrast, establishes a pointwise balance between the eigenvalues of the Hessian, $D^{2} u$. It is a purely infinitesimal relation, and the only passage from the infinitesimal to the local is given by the very imprecise Alexandrov Backelman Pucci theorem, that makes recourse of the Monge Ampere equation, simultaneously an extremal for second order equations and with a hidden divergence structure.

Lacking the weak Hilbert space formulation of divergence equation, we must view fully non-linear equations as a "restriction" of possible quadratic behaviors.

To "describe" a non-divergence equation, for instance $\Delta u=0$, it is enough to "list" all superharmonic and subharmonic quadratic polynomials.

That is, we "characterize" the surface

$$
\text { "Trace } M=0 "
$$

in the space of matrices implicitly by "listing" all matrices whose trace is positive or negative.

This is the way in which existence and regularity theory for fully non-linear equations is done (the Crandall-Lions viscosity method), and periodic homogenization is done.

Given an equation, say for simplicity:

$$
A_{i j}(x) D_{i j} u=0
$$

with periodic coefficients, and a global homogeneous profile, now a quadratic polynomial $P(x)$, you want to construct a " $P$-like" solution, $u_{0}$, of $L u_{0}=0$, that is contained in a strip around $P_{0}$. 
In general this is not possible: only selected polynomials will accept such a solution (the same way that pulsating waves will be "front-like" only for a very specific front that determines the "effective speed" of the wave). In other words, given

$$
P_{0}^{\lambda}=P_{0}(x)+\lambda|x|^{2},
$$

there is only one value $\lambda_{0}$ of $\lambda$ for which we are able to construct the corresponding $u_{0}$.

For larger $\lambda, P_{0}^{\lambda}$ would be a subsolution of the homogenized equation, for smaller $\lambda$ a supersolution.

Let me stress once more the difference: In the homogenization of divergence equations every linear function is a solution of the limiting equation and the "linear-like solutions"; the correctors are used to "calculate" the effective equation, both in the periodic and random case.

In the non-divergence case only "selected quadratic polynomials" admit a $P$-like solution and these define the effective equation

$$
F_{0}\left(D^{2} u\right)=0 \text {. }
$$

Let us now go back to our random equation

$$
F\left(D^{2} v, X, \omega\right) \text {. }
$$

As we discussed above, we assume that

a) $F$ is uniformly elliptic in $D^{2} v$, for every $X$ and $\omega$ (for instance, the sup of a family of uniformly elliptic linear operators).

b) Solutions are unique (Lipschitz regularity in $X$ is a sufficient condition).

c) As we said before $\omega$ indicates the random deformation that $F$ has suffered, and lives in a probability space $M, \mu$.

Of course, if we change coordinates by a translation, $X$ to $X+Y_{0}$, our deformations are still the same probability distribution although we may have labeled differently:

$$
\mathcal{T}_{Y_{0}}(\omega)
$$

That is

$$
F\left(D^{2} v, X, \omega\right)=F\left(D^{2} v, X+Y_{0}, \mathcal{T}_{Y_{0}}(\omega)\right)
$$

where we ask the transformation $\mathcal{T}_{Y_{0}}$ from $M$ to $M$ to measure preserving.

Finally, we expect some averaging at long distance. If $A \subset M$ is a set of positive probability, we expect

$$
\mu\left(\bigcup_{Y_{0} \in \mathbb{Z}^{n}} \mathcal{T}_{Y_{0}}\left(\mathbb{Z}^{n}\right)\right)=1
$$

(Ergodicity.) 
THEOREM 3. There exists a homogenization limit equation

$$
F\left(D^{2} u_{0}\right)=0
$$

to which solutions of the $\omega$ problem converge almost surely.

\section{Chess board}

$$
\begin{aligned}
& L_{1}=U_{x x}+U_{y y} \text { with prob } p \text { (white) } \\
& L_{2}=2 U_{x x}+U_{y y} \text { with prob 1- } p \text { (black) }
\end{aligned}
$$

Each array is an $\omega$

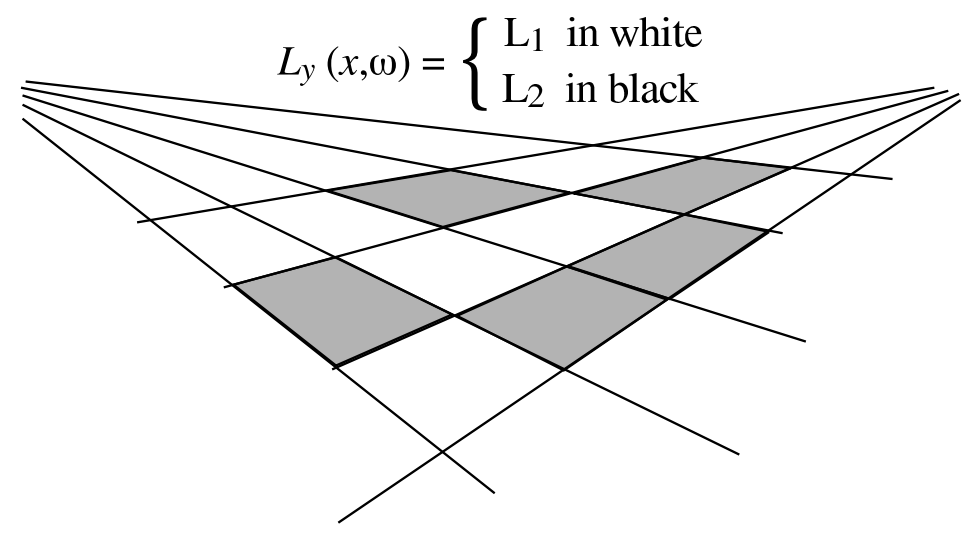

Figure 12.

\section{Main ingredients of the proof.}

Periodicity is replaced by the subadditive ergodic theorem: Let $\lambda(\theta, \omega)$ satisfy $0 \leq \lambda \leq|Q|$, be subadditive: If $Q=\cup Q_{j}$ (disjoint) then

and invariant

$$
\lambda_{Q} \leq \sum \lambda_{Q_{j}}
$$

$$
\lambda\left(Q\left(x-x_{0}, \omega\right)\right)=\lambda\left(Q(x), \tau_{x_{0}}(\omega)\right)
$$

then as $|Q|$ goes to infinity, $\frac{\lambda(Q, \omega)}{|Q|}$ converges to a constant $h_{0}$.

Following the lines of the periodic homogenization process, the idea is now to describe implicitly the equation $F\left(D^{2} u\right)=0$ by finding out which polynomials should be super- or subsolutions of the limiting equation.

That is, let us choose a polynomial $P_{0}$ in a large cube $Q_{M}$, and let us consider simultaneously $P_{0}+\lambda|x|^{2}$ and the solution $u$ to

$$
F_{\omega}\left(D^{2} u, x\right)=0
$$

sharing boundary data along $\partial Q$.

If $\lambda$ is very large, $P_{\lambda}=P_{0}+\lambda|x|^{2}$ is a subsolution of $L_{\omega}$ and thus

$$
P_{0}+\lambda|x|^{2} \leq u \text {. }
$$


Equally if $\lambda$ is very negative

$$
P_{0}+\lambda|x|^{2} \geq u \text {. }
$$

So, for some intermediate value of $\lambda, P_{0}+\lambda|x|^{2}$ and $u$ start crossing each other.

At that point, to force monotonicity in our process we substitute $u$ by $v$, the least supersolution above $P$.

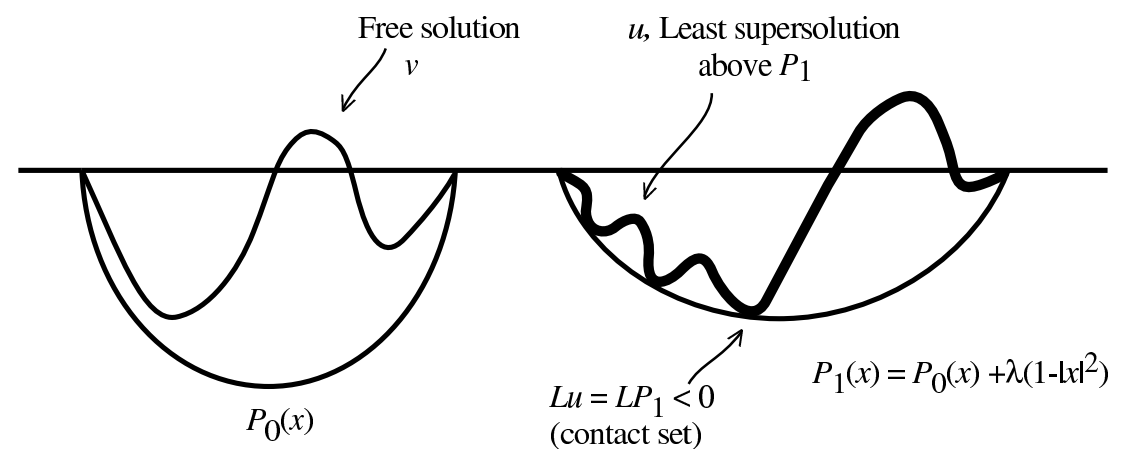

FiguRE 13.

Now let's forget the $\lambda$ and start again:

Choose a $P$, and for a given $\omega$ and $Q$ consider the function $v$, least supersolution of $L_{\omega} v=0$ in $Q$, above $P$. Such a $v$ satisfies

$$
L_{\omega}(v)=L_{\omega}(P) \chi_{v=u} \leq 0 .
$$

In fact $\left|\chi_{v=0}\right|$ controls $\|u-v\|$.

The uniform positive density of examples a) and b) is now replaced by :

If $v=P$ at $x_{0}, v$ separates quadratically (see Figure 14)

$$
|v-P| \leq C\left|x-x_{0}\right|^{2}
$$

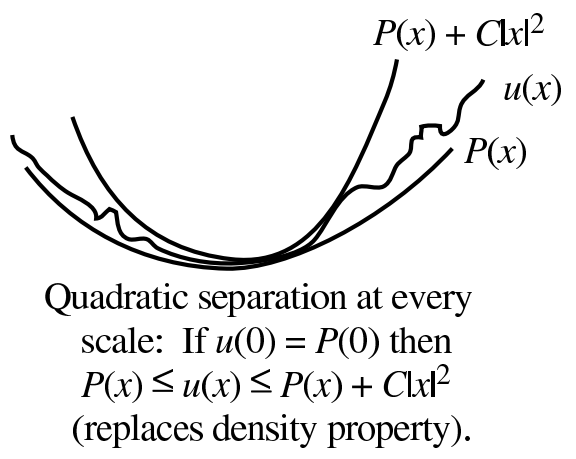

Figure 14. 
and the Birkhoff property by

$$
|\{v=P\}|=\lambda(Q, \omega)
$$

is a subadditive function of $Q$. In fact if $Q=\cup Q_{j}$,

$$
\left\{v_{Q}=P\right\} \subset \cup\left\{v_{Q_{j}}=P\right\} .
$$

Therefore $\frac{\lambda(Q)}{|Q|}$ has a limit $h$ as $|Q|$ goes to infinity.

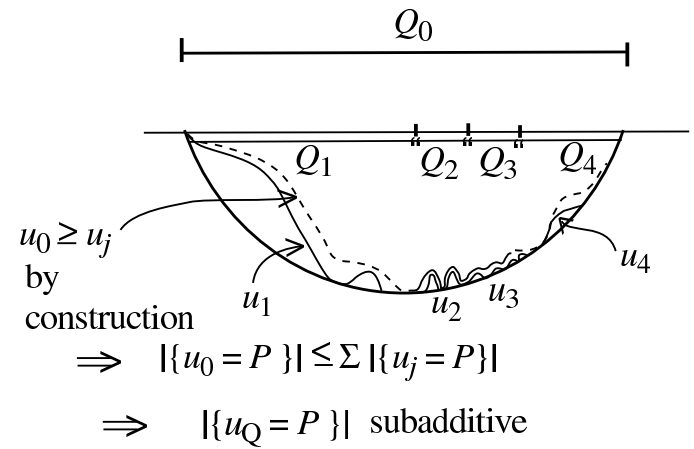

FiguRE 15.

If $h=0$, that means that $v$ at infinity decouples from $P$, and thus in the $\varepsilon$ rescaled limit the free solution $u$ (from $A-B-P$ estimates). That is $P$ has to be a subsolution of the "unknown" limiting equation. (See Figure 16.)

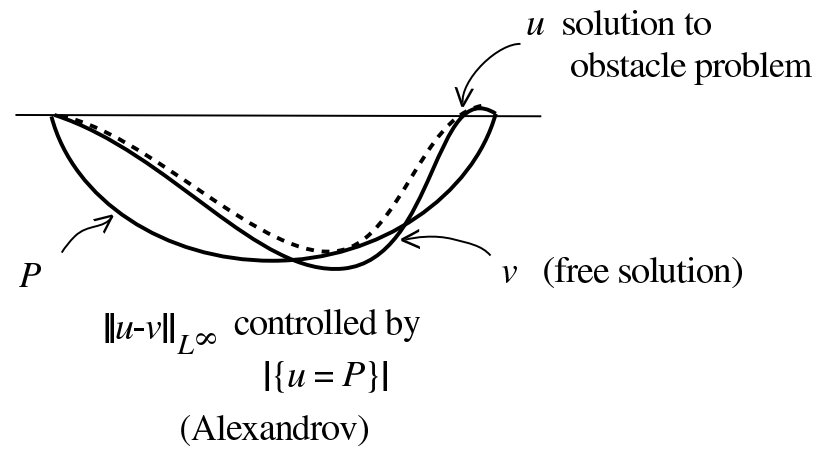

FiguRe 16.

If $h \neq 0$, from subadditivity this forces $\{v-P\}$ to spread all over $Q$ as $|Q|$ goes to infinity.

Indeed, if we divide $Q$ into, say, 64 equal subcubes $Q_{j}$, then $\frac{\lambda\left(Q_{j}\right)}{\left|Q_{j}\right|}$ is also converging to $h$, but since $\left\{v_{Q}=P\right\} \subset \cup\left\{v_{Q_{j}}=P\right\}$ that forces $\left\{v_{Q}=P\right\}$ to be spread all over $Q$. (See Figure 17.)

The quadratic growth estimate implies that in the ( $\varepsilon$ rescaled) limit $v_{Q}=P$ and thus $u \leq v_{Q}=P$. Therefore $P$ is a supersolution. This 


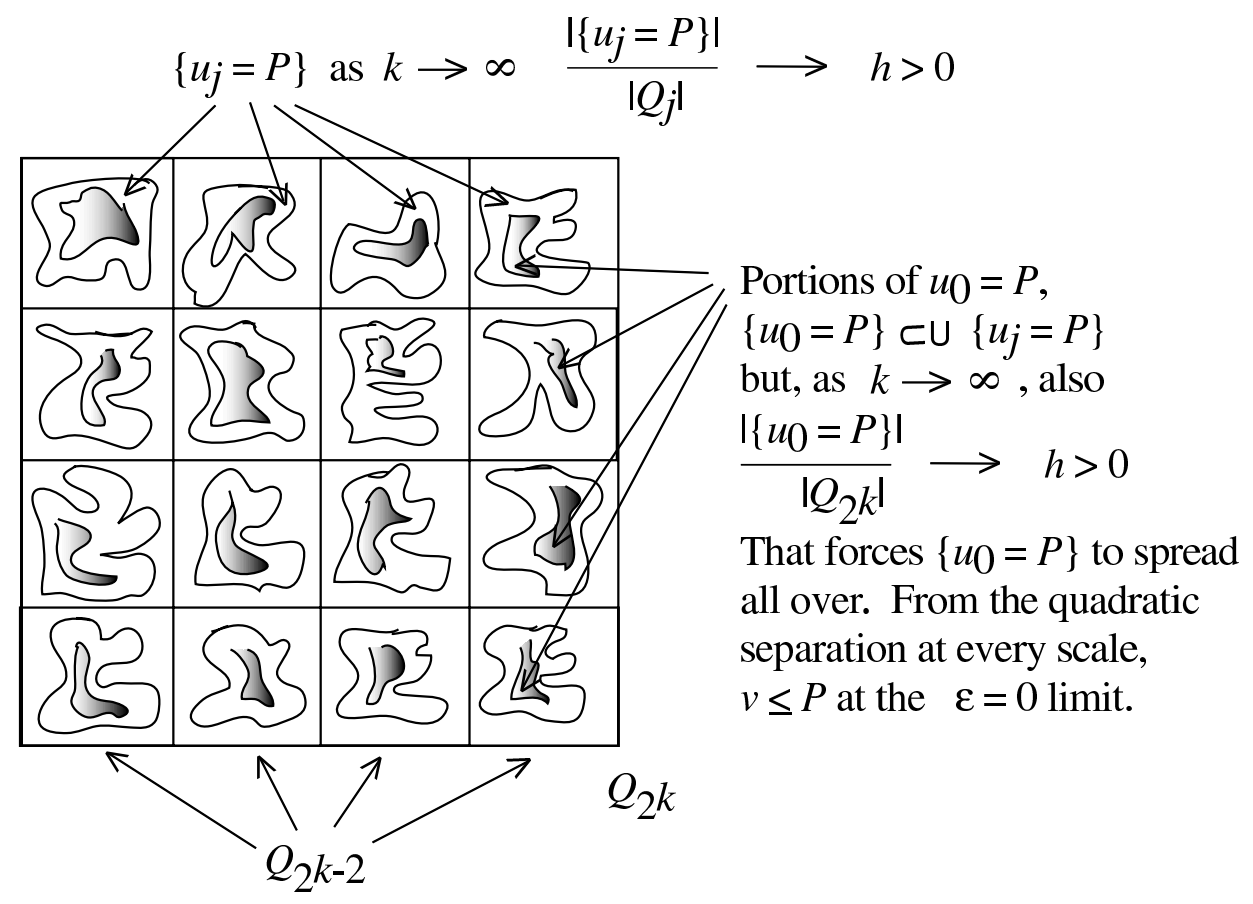

FiguRe 17.

describes implicitly the operator $A_{i j}$. The general homogenization limit theorem is then not hard to prove. See Caffarelli, Souganidis, Wang [13] for details and the extensive list of related articles and different approaches to random homogenization.

We conclude with several remarks.

As we have seen, surfaces evolving or embedded in a stationary fashion in a periodic medium exhibit non-uniqueness and hysteresis, and a Perron-like method provides the selected solution for an advancing or retreating process, as well as the desired monotonicity to treat random media.

To complete this section, a word on rates of convergence: Suppose that correlation of our "deformations" at far away points decays at a fast rate. In fact, let's suppose they are independent, i.e., each deformation "influences" a compact neighborhood of diameter $M$.

Then it is possible to use the methods above to prove in a quite straightforward fashion that solutions to

$$
F_{\omega}\left(D^{2} u, \frac{X}{\varepsilon}\right)
$$

converge to the homogenized limit a rate

$$
\varepsilon^{-\log \varepsilon}
$$


in the non linear case, and $\varepsilon^{t}$ for some $t$ in the linear (replicating in this case the results of Yurinskii).

This is done by estimating the rate at which the contact set between $u$ and $P$ from above or below, decays (Caffarelli-Souganidis, to appear).

This suggests a series of possible questions: Can we prove random homogenization for minimal surfaces or free boundaries in random media?

For pulsating waves, as pointed above the answer is yes for one dimension and for higher dimensions is a matter of several technical issues, but seems plausible.

For minimal surfaces the approach we used for fully non-linear equations suggests looking at the full foliation, i.e., minimize

$$
\int\left|\nabla u_{T} A_{i j}(x) \nabla u\right|^{1 / 2}
$$

with the constraint

$$
\langle e, x\rangle \leq u \leq M+\langle e, x\rangle
$$

with the hope that as $M$ goes to infinity the foliation becomes slowly "unconstrained". A correlation rate may be necessary. Can we attack with this method other random problems, like the Neumann problem? What is the correct Neumann problem we want to study?

\section{References}

[1] F. Auer, Minimale Hyperflächen in Riemannische n-Torus, Doctoral dissertation, Albert-Ludwigs Universität Frieburg, Freiburg, Germany, 1997.

[2] V. Bangert, Mather sets for twist maps and geodesics on tori, Aequationes Math. 34(2-3) (1987), 153-166.

[3] V. Bangert, Laminations of 3-tori by least area surfaces, Analysis, et cetera, 85-114, Academic Press, Boston, 1990.

[4] L.A. Caffarelli and R. De La Llave, Plane-like minimizers in periodic media, Comm. on Pure and Appl. Math. 54(12) (2001), 1403-1441.

[5] M. Gromov, Foliated Plateau problem. I. Minimal varieties, Geom. Funct. Anal. 1(1) (1991), 14-79.

[6] M. Gromov, Foliated Plateau problem. II. Harmonic maps of foliations, Geom. Funct. Anal. 1(3) (1991), 253-320.

[7] G. Hevlund, Geodesics on a two-dimensional Riemannian manifolds with periodic coefficients, Ann. of Math. 33 (1932), 719-739.

[8] H.M. Morse, A fundamental class of geodesics on any closed surface of genus greater than one, Trans. Amer. Math. Soc. 26(1) (1924), 25-60.

[9] J. Moser, Minimal solutions of variational problems on a torus, Ann. Inst. H. Poincaré Anal. Non Linéaire 3(3) (1986), 229-272.

[10] H. Berestycki, L.A. Caffarelli, and L. Nirenberg, Uniform estimates for regularization of free-boundary problems, Lecture Notes in Pure and Appl. Math., 122, Marcel Dekker, 1990, 567-619.

[11] H. Berestycki and F. Hamel, Front propagation in periodic excitable media, Comm. Pure Appl. Math., 2002. 
[12] L.A. Caffarelli, K.-A. Lee, and A. Mellet, Singular limit and homogenization for flame propagation in periodic excitable media, Arch. Rat. Mech. Anal., to appear.

[13] L.A. Caffarelli, P.E. Souganidis, and L. Wang, Homogenization of fully nonlinear uniformly elliptic equations, Comm. in Pure and Applied Mathematics 58 (2005), 319-361.

[14] F. Castell, Homogenization of random semi-linear PDEs, Prob. Theory Relat. Fields 121 (2001), 492-524.

[15] G. Dal Maso and L. Modica, Nonlinear stochastic homogenization and ergodic theory, J. Reine Angew. Math. 368 (1986), 28-42.

[16] G. Dal Maso and L. Modica, Nonlinear stochastic homogenization, Ann. Math. Pura Appl., 1985, 347-389.

[17] L.C. Evans, Periodic homogenization of certain fully nonlinear partial differential equations, Proc. Roy. Soc. Edinb. 120A (1992), 245-265.

[18] H. Ishii, Homogenization of the Cauchy problems for Hamilton-Jacobi equations, Stoch. Analysis Controls Optimization and Applications, 305-324, Systems and Control Foundations and Applications, Birkhäuser, Boston, 1999.

[19] S.M. Kozlov, The method of averaging and walks in inhomogeneous environments, Russian Math. Surveys 40 (1985), 73-145.

[20] P.-L. Lions and P.E. Souganidis, Homogenization for stochastic viscous Hamilton-Jacobi equations, preprint.

[21] P.-L. Lions and P.E. Souganidis, Correctors for the homogenization of HamiltonJacobi equations in a stationary ergodic setting, Comm. in Pure and Applied Math. 56 (2003), 1501-1524.

[22] G. Papanicolaou and S.R.S. Varadhan, Boundary value problems with rapidly oscillating random coefficients, Proc. Colloq. on Random Fields, Rigorous results in statistical mechanics and quantum field theory (J. Fritz, J.L. Lebaritz, D. Szasz, eds.), Colloquia Mathematica Societ. Janos Bolyai 10 (1979), 835-873.

[23] G. Papanicolaou and S.R.S. Varadhan, Diffusion with random coefficients, Essays in Statistics and Probability (P.R. Krishnaiah, ed.), North Holland Publishing Company, 1981.

[24] F. Rezankhanlou and J. Tarver, Homogenization for stochastic Hamilton-Jacobi equations, Arch. Rat. Mech. Anal. 151 (1999), 1-11.

[25] P.E. Souganidis, Stochastic homogenization of Hamilton-Jacobi equations and some applications, Asympt. Anal. 20 (1999), 1-11.

[26] V.V. Yurinskii, Averaging of symmtric diffusion in random medium, Siberian Math. J. 27 (1986), 167-180.

[27] V.V. Yurinskii, Homogenization error estimates for random elliptic operators, Lectures in Appl. Math., 27, Amer. Math. Soc., Providence, RI, 1991.

Department of Mathematics, The University of Texas at Austin, Austin, TX 78722

E-mail address: caffarel@math.utexas.edu 
